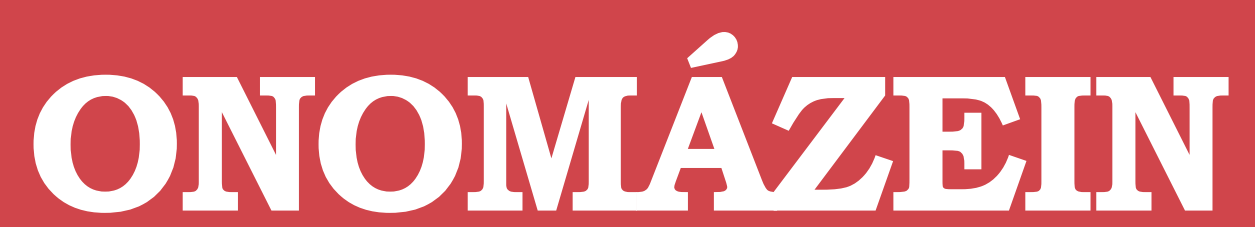

Revista semestral de lingüística, filología y traducción
PONTIFICIA UNIVERSIDAD CATÓLICA DE CHILE CATOLICA DE CHILE
FACULTAD DE LETRAS

\title{
Condensation: A translation device for revealing complexity of knowledge practices in discourse, part 2-clausing and sequencing
}

\author{
Karl Maton \\ The University of Sydney \\ Australia
}

\author{
Y. J. Doran \\ The University of Sydney \\ Australia
}

Número especial
ONOMÁZEIN Número especial LSF y TCL sobre educación y conocimiento (2017): 77-110 DOI: 10.7764/onomazein.sfl.04

\section{(c) $\underset{\mathrm{BY}}{(\mathrm{ND}}$}

Karl Maton: LCT Centre for Knowledge-Building, The University of Sydney, Australia.

| Correo electrónico: karl.maton@sydney.edu.au

Y. J. Doran: Department of Linguistics, The University of Sydney, Australia.

| Correo electrónico: yaegan.doran@sydney.edu.au

Fecha de recepción: 2 de octubre de 2015

Fecha de aceptación: 29 de enero de 2016 


\section{Abstract}

Complexity of knowledge practices is undertheorized in education research because knowledge is often conceived cognitively. Legitimation Code Theory conceptualizes this complexity in terms of 'semantic density', which explores how meanings are interrelated within practices. This concept is becoming widely enacted in research, a flexibility that raises the question of identifying 'semantic density' in specific objects of study. This is the second of two papers that offer a 'translation device' for identifying 'epistemic-semantic density' (where condensed meanings are formal definitions or empirical descriptions) in English discourse. The first paper (this issue) provided tools for exploring how individual words reveals different strengths of epistemic-semantic density. Those concepts revealed different degrees of complexity of knowledge. This paper outlines tools for exploring how the ways actors combine words reveals 'epistemological condensation' or strengthening of epistemic-semantic density. It provides typologies for identifying different kinds of 'clausing' and 'sequencing' and describes how these types manifest varying degrees of increasing complexity. These concepts reveal different kinds of knowledge-building. Two contrasting examples, from a secondary school History classroom and a scientific research article, are analysed to illustrate the insights into complexity offered by the tools outlined in both papers.

Keywords: Legitimation Code Theory; semantic density; translation device; language of description; knowledge-building, complexity. 


\section{Introduction}

This paper explores how knowledge-building can be explored through analysing the discourse used by actors ${ }^{1}$. It is the second of two papers on the complexity of knowledge practices. We strongly recommend reading its prequel, entitled 'Semantic density' (henceforth 'Paper I') before the current paper: much of what follows assumes understanding of previously outlined concepts and limits of space preclude adequate summaries here. It is merely as an aide-memoire that we shall outline some key issues from Paper 1 before introducing the current paper.

In Paper 1 we argued that the complexity of knowledge practices has been undertheorized because most research obscures knowledge as an object. We introduced the concept of semantic density from Legitimation Code Theory (LCT) as a means of understanding complexity and focused on one form, epistemic-semantic density, based on epistemological condensation of formal definitions and empirical descriptions ${ }^{2}$. We highlighted the applicability of this concept to diverse problem-situations and the corresponding diversity of its empirical realizations. This flexibility, we argued, underlines a need for 'translation devices' that make explicit how the concept is enacted within objects of study. We then introduced a translation device for English discourse, comprising four sets of typologies for exploring:

- strengths of epistemic-semantic density at the level of words (wording and word-grouping tools); and

- degrees of epistemological condensation (or strengthening of epistemic-semantic density) by combining words into short passages (clausing tool) and by combining short passages into longer sequences of discourse (sequencing tool).

In Paper 1 we defined the wording and word-grouping tools and illustrated how they may reveal complexity in the knowledge being expressed. In this paper we introduce the clausing and sequencing tools and illustrate how they may reveal signs of building complexity in knowledge. We are thus shifting focus from individual words to how words are combined, from states of complexity to processes creating complexity, and from forms of knowledge to knowledge-building. These accompany a conceptual shift from epistemic-semantic den-

1 We are grateful to Talia Gill for contributing to earlier versions of tools. This paper arises from the PEAK Project, supported by the Australian Research Council (Discovery Project, DP130100481).

2 'Semantic density' is from the Semantics dimension of LCT. See Maton (2011a, 2011b, 2014a, 2014b, 2016a, 2016b) and Martin \& Maton (this issue) for introductions; and see Blackie (2014), Clarence (2014), Doran (this issue), and Macnaught et al. (2013) for examples of enactment in research and praxis. 
sity (henceforth 'ESD') to epistemological condensation or differences in the strengthening of ESD. Epistemological condensation describes a continuum from lower epistemological condensation (EC-) or less strengthening to higher epistemological condensation (EC+) or more strengthening. We must emphasize: we are focused wholly on addition of meaning, the difference is how much. Though denoted by a minus sign, even lower epistemological condensation involves addition; 'EC-' means 'adding relatively fewer meanings'. Thus we describe 'higher/lower' rather than 'stronger/weaker', as the latter might suggest the removal of meanings ${ }^{3}$.

\subsection{The nature of the tools}

Paper 1 explained the nature of a translation device. Space precludes repeating that discussion, though five attributes are worth emphasizing to avoid misreadings. First, the tools translate between epistemological condensation (not axiological condensation of affective, aesthetic, ethical, political or moral stances) and English discourse (not other languages or semiotic resources). In addition, they explore different kinds of word combinations and not their ontological referents; when discussing a taxonomy or process, we conceptualize their description in discourse and not the taxonomy or process itself.

Second, the tools are for translating sociological concepts by 'reading' discourse for signs of increasing complexity of knowledge practices. They are not taxonomies of clauses or sequences per se. They do not conceptualize English discourse; they conceptualize how epistemological condensation may be revealed in English discourse.

Third, the tools are practical: they help scholars allocate clausing and sequencing to locations along a continuum from higher to lower epistemological condensation. Empirical characteristics that we highlight as potential signs of types are thus only 'rules of thumb' and we intentionally use such terms as 'likely' and 'typically'.

Fourth, as illustrated in Figure 1, the typologies were created through recursive division. We searched for the most salient and robust basis for dividing clausing or sequencing into higher and lower epistemological condensation, then for a basis of dividing up each

3 We are not concerned with epistemological rarefaction, the removal of epistemic meanings (Maton, 2014b: 130). Preliminary analysis suggests this is often stated explicitly in English discourse (such as 'This does not mean ...'), though future work may explore subtler forms. Moving from stronger to weaker ESD in wording does not necessarily indicate rarefaction; for example, paraphrasing a technical into an everyday shifts from stronger to weaker ESD wording but itself adds meaning to the unfolding discourse. 
of those two types into higher and lower, and so on (see Paper 1). Each sub-type is thus approached through its overarching type, a hierarchy highlighted by differences in the horizontal lines.

Finally, three general features of ESD outlined in Paper 1-relationality, differentiation and resonance-remain relevant to epistemological condensation. For wording, a key indicator of ESD is the number of relations meanings enjoy with other meanings. For clausing and sequencing, a key indicator of epistemological condensation is the number of new relations established among meanings. Typically, the more relations created, the greater the likely differentiation of referents and the more resonance meanings are likely to have with other related but unspecified meanings. Thus, the wording tool highlights the complexity of epistemological constellations within which words are located, and the clausing and sequencing tools highlight the increase in complexity of those constellations.

We shall now introduce these concepts. Section 2 outlines the clausing tool and section 3 outlines the sequencing tool. Section 4 illustrates their potential usefulness through analyses of the same examples discussed in Paper 1 : a secondary school History lesson and a scientific research article.

\section{Clausing}

The clausing tool is summarized in Table 1. By 'clausing' we mean combining words (or word-groupings) into a short, coherent passage capable of standing alone in a way that changes their ESD ${ }^{4}$. The italicized words are essential: we are not concerned with a unit of language per se but with identifying short, stand-alone passages involving epistemological condensation. Thus, the tool outlines types of clausing that strengthen ESD of words (and word-groupings) to different degrees.

The tool offers three levels of delicacy. Section 2.1 introduces two principal types: connecting and augmenting. Section 2.2 discusses taxonomizing and coordinating forms of connecting; and section 2.3 discusses characterizing and establishing forms of augmenting. Both sections also explore a further level of delicacy that distinguishes within each of these subtypes. As illustrated here, names of clausing categories are always italicized to avoid confusion with other possible meanings.

4 'Word-grouping' strengthens the ESD of words without generating coherent passages capable of standing alone (e.g. 'The five particles in physics are..., compared to the clausing of 'In physics, there are five particles'). To create an appliable device, we considered word-grouping part of identifying the ESD revealed by words (Paper 1 ). 


\section{FIGURE 1}

Recursive division of epistemological condensation from a continuum into categories

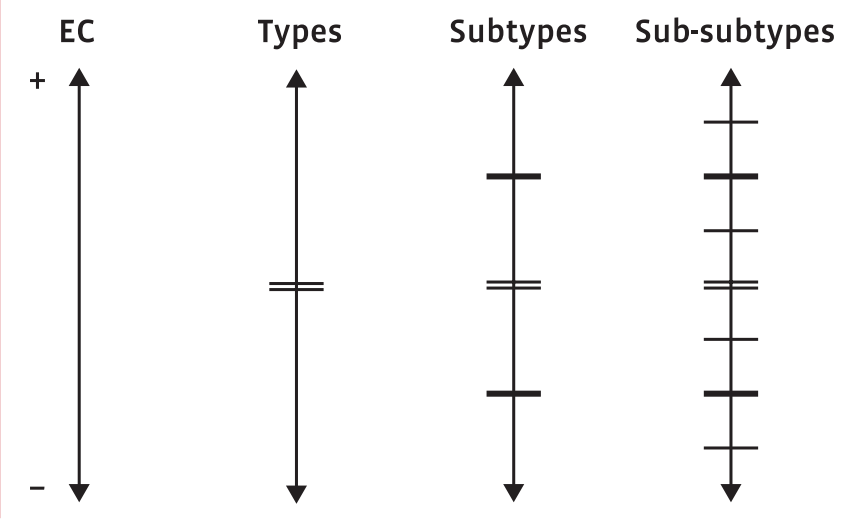

\section{TABLE 1}

Clausing tool for epistemological condensation

\begin{tabular}{|c|c|c|c|}
\hline EC & Type & Subtype & Sub-subtype \\
\hline \multirow{7}{*}{+} & \multirow{4}{*}{ connecting } & \multirow{2}{*}{ taxonomizing } & classifying \\
\hline & & & composing \\
\hline & & \multirow{2}{*}{ coordinating } & causing \\
\hline & & & correlating \\
\hline & \multirow{4}{*}{ augmenting } & \multirow{2}{*}{ characterizing } & displaying \\
\hline & & & dramatizing \\
\hline & & \multirow{2}{*}{ establishing } & positioning \\
\hline$\underline{\downarrow}$ & & & positing \\
\hline
\end{tabular}

\subsection{Clausing types}

The first level of delicacy explores whether a short passage explicitly locates meanings within complex epistemological constellations of meaning, such as the schemes of classification, composition, causation and correlation associated with specialized domains of social practice (academic disciplines, professions, trades, etc.). This distinguishes between:

- connecting (EC+) that relates terms to epistemological constellations; and

- augmenting (EC-) that adds meanings to terms themselves without relating them to such constellations. 
Connecting is likely to generate higher epistemological condensation because constellations already include a number of meanings. Plugging a term into a constellation thereby simultaneously creates relations with all its members. For example, adding a type to a typology creates new relations with each existing type. Similarly, 'the vertebrate heart is principally composed of cardiac muscle and connective tissue' connects the underlined terms into a (compositional) taxonomy. Each term is not only related to the other two but also resonates out to unspecified meanings already related to those terms, such as taxonomies associated with 'cardiac muscle' (e.g. its composition of cardiomyocytes), 'connective tissue' (including its components of extrafibrillar matrices) and 'the vertebrate heart' (such as constituting a key component of the cardiovascular system). The compositional schema thus connects three compositional schemas (and more besides). Similarly, 'differences in member contexts cause differences in repertoires' connects two existing sets of meanings. Thus, by relating knowledge to already complex knowledge, connecting considerably strengthens its ESD.

Augmenting does not connect words into epistemological constellations but rather adds meanings directly to words themselves. Thus augmenting is less likely to simultaneously add multiple relations to other meanings. The song lyric 'the Queen is dead', for example, gives 'the Queen' the quality of being 'dead' but does not connect 'the Queen' into an epistemological constellation. Though 'the Queen' and 'dead' can be connected into explicit schemas such as taxonomies or processes such as causation, this is not the case here. Thus 'the Queen is dead' has little impact on the quality of being dead; any epistemic meanings related to 'dead' are little changed and its subsequent use resonates little with epistemic meanings related to 'The Queen'. Similarly, 'the strong nuclear force has a short range' augments 'the strong nuclear force' with the distance to which it has an effect but does not connect it to, for example, a taxonomy of types of force. Thus, while adding meaning to words, augmenting represents relatively lower epistemological condensation.

The distinction between connecting and augmenting concerns the number of relations being created among meanings. However, not all such relations are equal: some enable more epistemological condensation than others. We can thus distinguish within each type according to the forms taken by connecting (section 2.2) and by augmenting (section 2.3).

\subsection{Subtypes of connecting}

As shown in Table 1, two kinds of connecting can be distinguished according to the forms taken by the connections they establish:

- taxonomizing that definitionally connects terms in definite relations of an ordered schema; and

- coordinating that connects terms in contingent relations of causation or correlation. 
Both are subtypes of connecting and so offer relatively high epistemological condensation. However, of the two, taxonomizing generates higher condensation because its definitional connections enable both more meanings to be related and those relations to be more interlocking.

Taxonomizing typically creates classificatory 'type-subtype' relations or compositional 'part-whole' relations. These connect terms in ways that condense each term with meanings from the others by definition. For example, in 'Bernstein sets out two types of knowledge structure: hierarchical knowledge structures and horizontal knowledge structures', each of the two types of 'knowledge structure' are condensed with its meanings and, conversely, 'knowledge structure' is condensed with the notion there are two types with distinctive features. In addition, 'type-type' relations are elaborated between 'hierarchical' and 'horizontal', highlighting they both share meanings (as 'knowledge structures') and possess distinctive meanings (as different types). There is thus considerable cross-condensation of meanings among terms and, moreover, these meanings are 'locked in' through definition, creating definite and necessary relations.

In contrast, coordinating connects meanings in contingent relations of effect: changes in referents of one term are held to involve changes in those of another. For example, 'the number of spectral lines increases with the n value' connects the two underlined clusters, creating multiple relations among meanings. This offers relatively high epistemological condensation but lower than taxonomizing because meanings are not cross-condensed through definitions. The example does not state that 'the number of spectral lines' is a kind of or a part of 'the $n$ value'. Coordinating may posit ' $X$ changes with $Y$ ' or ' $X$ causes $Y$ ' but $Y$ does not partake of the meanings of $X$. To share characteristics, one needs to rewrite the example into taxonomizing, such as: 'The increase in spectral lines is part of a process involving the $n$ value'5. Thus, coordinating generates high epistemological condensation through elaborating constellations but the resulting connections are more contingent than taxonomizing as they do not share essential characteristics among members.

\subsubsection{Finest level of connecting}

A third level of delicacy distinguishes further forms of connecting within taxonomizing and within coordinating (see Table 1).

5 By describing a process of correlation using both coordinating and taxonomizing we illustrate here that no set of referents is necessarily associated with a specific category of clausing. The tool translates between ESD and English discourse, not the referents of that discourse (section 1.1). 
First, taxonomizing can be distinguished into:

- classifying that creates classificatory relations of type-subtype or type-type; and

- composing that creates compositional relations of part-whole or part-part.

Classifying generates the highest epistemological condensation of clausing by developing a classificatory taxonomy. In its strongest form, classifying equates different terms as the same type, such as: 'Here intensity means brightness'; and 'This process of coating to enhance phagocytosis is called opsonization'. In such equating all meanings of each term are cross-condensed, maximizing shared meanings and 'locking in' those meanings by definition. Another, slightly less strong form describes one term as a subtype of another. In this subtyping meanings associated with the higher-order type are necessarily adopted by the lowerorder subtype (though not vice versa) because for $X$ to be a subtype of $Y$ it must possess the relevant features of $Y$ (in addition to its own distinctive features). Thus, not only is the subtype connected to a complex constellation (the typology) but meanings are also definitionally condensed from the type. For example, 'the proton is a baryon' both connects 'the proton' to a typology of subatomic particles, differentiating it from others such as mesons and leptons, and condenses meanings of 'baryon' (such as possessing three quarks) into the term'.

Composing describes one thing as part of another thing, creating a part-whole taxonomy. For example, in 'the heart contains two atria and two ventricles', all constituent components of the 'two atria' and the 'two ventricles' are placed within the taxonomy involving 'the heart'. This generates relatively high epistemological condensation but, unlike classifying, the lower-order 'part' does not share all the meanings of their higher-order 'whole'. Although 'two atria' and 'two ventricles' share meanings (and part-part relations) concerning composition of 'the heart', they are not themselves necessarily condensed with all its features. While 'the heart' includes meanings associated with both receiving blood from veins and expelling blood through arteries, each part involves meanings concerning only one or the other. Moreover, whereas in classifying the subtype is an example of the type (protons are examples of baryons), in composing this is not the case (a ventricle is not a heart). Thus, composing does not reach the heights of epistemological condensation of classifying.

Second, coordinating can be distinguished into:

- causing that specifies a causal relationship; and

- correlating that indicates a correlation or dependency but without specifying its nature.

6 Equating and subtyping are two of many examples in these papers of the potential for further levels of delicacy, should problem-situations require them. 
As forms of coordinating, both causing and correlating connect terms in contingent relations of effect where changes in one involve changes in the other(s), rather than sharing intrinsic characteristics among terms. However, of the two, causing generates higher epistemological condensation because it posits not only that one term is in some way dependent on another (as correlating does) but also differentiates the specific form taken by relations between them. It adds the meaning that ' $X$ causes $Y$ ' to ' $X$ is related to $Y$ '. For example, in 'pathological myopia is linked with functional blindness', the two terms are correlated but its nature is unspecified; in contrast, 'pathological myopia causes functional blindness' adds that the relation is causal. Thus causing offers higher epistemological condensation than correlating.

\subsection{Subtypes of augmenting}

Both principal types of clausing_connecting and augmenting_add meanings to words. While connecting elaborates epistemological constellations, enabling rapid knowledge-building, augmenting adds meanings to terms directly, building knowledge at a more sedate pace. Nonetheless, augmenting also takes a variety of forms that we shall outline, beginning each time from the lower category in Table 1.

Augmenting can first be distinguished into:

- establishing the existence of terms; and

- characterizing the properties or actions of terms.

Establishing generates few (if any) relations between a term and others. For example, 'there is a light' simply establishes the existence of 'a light'. Similarly, 'For equation number 25 there are finite solutions only for integer values of l' proposes there exist 'finite solutions' for 'integer values of ' ' in 'equation number 25'. This does not place each term in a taxonomy, specify that solutions change with a change in other terms, or describe them. It merely establishes the existence of 'finite solutions' in this situation. Establishing thereby creates an unconnected node of meaning or 'Gwiffly' (see Paper 1). This node may subsequently become part of a constellation but as yet remains unrelated to other meanings. As our examples suggest, establishing in English often involves the words 'there is/are/were/etc.' or, where 'there' is absent (e.g. 'at the end of the platform are some stairs'), the word could be inserted ('at the end of the platform there are some stairs').

Characterizing generates higher epistemological condensation than establishing by attributing properties or actions to terms whose existence is assumed, thereby relating meanings to other meanings. For example, 'the valeur of any feature is entirely differential' adds more meanings than simply establishing the underlined phrase by indicating a trait that will resonate through further uses of the term. Similarly, in 'this derivation develops new relations 
among technical words', the existence of the underlined terms is assumed and they are engaged in the action 'develops', augmenting their meanings.

\subsubsection{Finest level of augmenting}

A third level of delicacy distinguishes further forms of augmenting within establishing and within characterizing (see Table 1).

First, establishing can be distinguished into:

- positing that establishes the existence of a term but nothing more; and

- positioning that establishes a term's existence within a particular set of meanings?

Positing establishes a solitary node of meaning or 'Gwiffly's. Repeating an earlier example, 'there is a light' (where 'there' is not specifying a location) posits the existence of 'a light' but does not relate it to other terms. Positing is thus the limiting case of establishing. When considered in a wider context, the meaning may not be isolated; as part of unfolding discourse its relevance to a constellation of meanings may be inferred from other sources (such as its inclusion). Nonetheless, in terms of epistemological condensation, by establishing a meaning without relations positing represents the lowest clausing category.

Positioning generates slightly higher epistemological condensation by proposing not only that a term exists but also that it relates to other terms, but without clarifying the nature of those relations. Typically, positioning identifies a location or time. For example, 'within the image, there remains a certain dependence on relations to the material world' indicates not only that the underlined phrase exists but that it does so within images. However, its specific relations to images is not made explicit. Similarly, 'for each / there are $2 /+1$ possible values of mi indicates that within a cluster of meanings denoted as 'each l' there exists ' $2 l+1$ possible values of mi but not how they relate. It thus generates slightly higher epistemological condensation than positing, though still relatively low.

Second, characterizing can be distinguished into:

- dramatizing actions, thoughts or speech; and

7 It should go without saying that we are not discussing ontology or epistemology.

8 In Paper 1 we used positing to introduce the notion of Gwiffly ('there is a Gwiffly') and began building a constellation through classifying two subtypes ('A' and 'B') and displaying their respective properties (red and blue). 
- displaying properties, qualities, attributes or traits.

Dramatizing specifies that a term is involved in a physical, verbal or mental action. This augments the term by indicating it can undertake the action but does not imbue the term with meanings from the action itself-it shows $X$ can do $Y$ but not that $X$ shares characteristics with $Y$. Thus, while creating relations among terms, dramatizing does not enable considerable sharing of meaning. For example, 'the digestion contents of the phagolysosome are eliminated' relates the underlined phrase to being 'eliminated' but that action is not here condensed into characteristics of 'the digestion contents...' Similarly, 'Pliny wrote Tacitus a letter', relates three (underlined) terms to the action 'wrote' as performing different roles. However, it does not by itself suggest they share epistemic meanings beyond that involvement. For example, it denotes that 'Pliny' wrote but does not explicitly imbue 'Pliny' with the meanings of 'writing'. Thus, in dramatizing actions are portrayed as relatively transitory events rather than the lasting qualities, attributes or traits denoted by displaying (see below); they are less tightly related to terms and thereby offer lower epistemological condensation?.

Displaying represents the highest epistemological condensation of forms of augmenting. Displaying describes the qualities, attributes or traits of terms, thereby adding relations to these properties. Unlike dramatizing, displaying not only creates relations but also enables meanings to be shared along those relations. For example, in 'the mercury atom is relatively big', the meanings of 'relatively big' are condensed into 'the mercury atom'. However, this flow of meanings is likely to be in one direction. For example, 'the Queen is dead' condenses 'the Queen' with the property of being dead but is less likely to condense the property of being dead with epistemological meanings from 'the Queen'. Thus, while the displayed term is likely to experience epistemological condensation, the displaying term is less likely to do so. Displaying is often (though not exclusively) achieved through adjectives; for example, "life is very long' portrays a property of life as its length, augmenting the word 'life' with a relation to the word-group 'very long'.

\section{Sequencing}

Though the clausing tool begins to show how building complexity in knowledge is expressed through discourse, clausing is not the limits of discourse. Speech and writing bring together short passages to create stretches of discourse which are, in turn, brought together into lon-

9 Dramatizing can be graded according to the number of terms associated with the action; for example: (1) 'The digestion contents of the phagolysosome are eliminated'; (2) 'We can note the attributive structures'; (3) 'Pliny wrote Tacitus a letter'; etc. 
ger stretches. The sequencing tool explores how this larger process can be 'read' for signs of building complexity in knowledge. The tool, summarized in Table 2, allows different forms of sequencing to be assigned to different strengths of epistemological condensation. Thus, we are again concerned not with a unit of language per se but rather with signs of strengthening ESD. The sequencing tool currently represents the macro level of the translation device, reaching from two short passages to unlimited stretches of discourse. For consistency of exposition, however, we shall primarily illustrate categories with sequencing of successive passages.

\section{TABLE 2}

Sequencing tool for epistemological condensation

\begin{tabular}{|c|c|c|c|}
\hline EC & Type & Subtype & Sub-subtype \\
\hline \multirow{7}{*}{$\stackrel{+}{\hat{\top}}$} & \multirow{4}{*}{ cumulative } & \multirow{2}{*}{ vertical } & integrative \\
\hline & & & subsumptive \\
\hline & & \multirow{2}{*}{ horizontal } & consequential \\
\hline & & & sequential \\
\hline & \multirow{4}{*}{ segmental } & \multirow{2}{*}{ sedimental } & reiterative \\
\hline & & & repetitive \\
\hline & & \multirow{2}{*}{ compartmental } & coherent \\
\hline- & & & incoherent \\
\hline
\end{tabular}

\subsection{Sequencing types}

The first level of delicacy explores whether sequencing adds meanings by explicitly linking passages. This distinguishes between:

- cumulative sequencing (EC+) that explicitly relates terms from different passages and so adds meaning; and

- segmental sequencing (EC-) that does not explicitly relate terms from constitutive passages.

Where segmental sequencing is (roughly) the sum of its parts, cumulative sequencing creates more than the sum of its parts and so enables higher epistemological condensation.

Cumulative sequencing relates passages in two main ways: 'horizontally' by linking passages conjunctively ('because', 'if...then', 'therefore', etc.) or 'vertically' by condensing meanings from one or more passages into terms located in another passage. In both cases, these relations generate meanings additional to those offered in the passages themselves. Consider, for example: 
The display of human remains on the site of Pompeii has not become a major issue even though the question of the treatment of skeletal remains has evoked impassioned debate in other parts of the world.

In this case 'even though' links the passages and adds that the first was unexpected given the second and that a different occurrence was expected, resonating out to other, unspecified events. A second example is 'vertical':

Vesicles in the cytoplasm called lysosome fuse with the phagosome releasing digestive enzymes such as lysozyme and proteases into the phagosome. The result of this fusion is called phagolysosome.

Here 'this fusion' condenses the underlined terms and relates their meanings to 'phagolysosome', generating more than the sum of the passages. This holds both ways: read in reverse, 'phagolysosome' is related via 'this fusion' to a series of meanings. Replacing the underlined terms with letters - $A$ in $B$ called $C$ does $D$ with $E$-reveals the complexity of what is condensed and related to a further term. Moreover, each term is a member of other constellations, generating further condensation, resonance to more meanings, and greater differentiation as relations are categorically specified. Cumulative sequencing thereby suggests rapid building of complexity in the knowledge being expressed.

In contrast, segmental sequencing generates lower epistemological condensation by not explicitly linking passages. This is particularly evident where passages appear unrelated, such as 'The heading there is "Limitations of the Bohr model". Okay, who's away?', which shifts focus from science knowledge to classroom management. However, it may involve passages on the same topic. For example, 'They don't have windows. They have very small slits' builds knowledge: the second passage develops the first by indicating what they have instead of windows. However, the way they are combined (without explicit linking) does not itself add further meanings. Thus, segmental sequencing expresses knowledge-building of a slower pace.

\subsection{Subtypes of cumulative sequencing}

As mentioned above, cumulative sequencing links passages in two main ways that represent distinct subtypes:

- vertical sequencing condenses meanings from one or more passages and transports those meanings into another passage; and

- horizontal sequencing links passages together without explicitly transporting meanings across passages. 
Vertical sequencing selects terms, packs their meanings into another term, and places that term into relations with further terms. Meanings are gathered 'upwards' from their original passage(s), condensed, transported to another context and recontextualized 'downwards' into a new passage. Consider, for example:

Systemic functional linguistics stratifies the content plane into lexicogrammar and discourse semantics. Stratification of this kind can be usefully compared with expansions of size and abstraction in other models.

The underlined passage is condensed into the word-group 'stratification of this kind' and related to 'expansions of size and abstraction in other models' in the second passage. Creating a term for transporting ESD in this way offers considerable epistemological condensation: the transporter contains multiple relations among a cluster of meanings that can be simultaneously related to further meanings. Here, 'stratification of this kind' condenses relations among 'systemic functional linguistics', 'content plane', 'lexicogrammar' and 'discourse semantics' into a transporter which itself adds meanings: 'stratification' is modified by 'of this kind' to resonate out to a taxonomy of types of stratification. One indicator of vertical sequencing can be words such as 'this', 'that', 'these', etc., referring to condensed terms ${ }^{10}$. This can be seen in the text you are reading: in the previous passage the bold word vertically sequenced its underlined predecessor. Another potential indicator can be consolidation of happenings into things, such as 'fuse' into 'fusion', 'stratifies' into 'stratification', etc.

Where vertical sequencing condenses and transports meanings, horizontal sequencing links passages. Though lacking a transporter means horizontal sequencing offers lower epistemological condensation than vertical sequencing, it is still relatively high because the links both create relations among passages and add meanings of their own. For example:

There is nothing wrong with going back to your Year 11 information on bath houses, because the activities that happened in one bath house generally happened in every other bath house.

In bringing these passages together in this way, a History schoolteacher relates their meanings, making clear that ideas discussed in Year 11 are generalizable, and adds meaning by specifying causal relations ('because') between the passages. This both adds relations and differentiates their form. As the example suggests, horizontal sequencing often involves linking words such as 'then', 'because', 'or', 'if', 'so', 'however', 'also', 'moreover', etc. As we discuss in section 3.2.1, the meanings these links add may include temporal sequence (e.g. 'and then'),

10 Where referring to a short passage, these words may indicate vertical sequencing, but where referring to a single word or word-group they may indicate a proxy (see Paper 1, section 3.4). 
cause (e.g. 'because', ‘so'), condition (e.g. 'if/then’), and counter-expectation (e.g. 'even though').

Vertical sequencing is likely to signal faster knowledge-building than horizontal sequencing, because condensing relations among meanings into transporter terms enables those multiple meanings and relations to be simultaneously related to further meanings. The two forms can also be used together to generate even higher epistemological condensation; for example:

From the perspective of discourse semantics, these relationships can be construed as structural, however this construal rests on clarifying how discourse structures differ from grammatical ones.

Here vertical sequencing (underlined-bold) condenses a series of meanings into 'this construal' at the same time as horizontal sequencing (italics) conjunctively links the passages. These interact to further elaborate meanings in each passage: vertical sequencing condenses the first passage and relates it to meanings in the second passage; horizontal sequencing adds meaning of counter-expectancy and ensures the sequence resonates out to other, unspecified meanings.

\subsubsection{Finest level of cumulative sequencing}

As shown in Table 2, a third level of delicacy can be discerned. First, vertical sequencing can be distinguished into:

- integrative sequencing that condenses and transports meanings from more than one passage; and

- subsumptive sequencing that condenses and transports meanings from within a single passage.

Both forms involve meanings being gathered together, condensed and relocated, but on different scales. Subsumptive sequencing often involves successive passages. Returning to this example:

Vesicles in the cytoplasm called lysosome fuse with the phagosome releasing digestive enzymes such as lysozyme and proteases into the phagosome. The result of this fusion is called phagolysosome.

A series of related meanings from one passage are subsumed into a new term. As here, this typically results in single terms, often involves consolidation of wording (e.g. 'fuse' becomes 'fusion'), and may include variations of 'this' to identify the meanings being subsumed. In 
contrast, integrative sequencing embraces longer stretches of discourse that include multiple passages and possibly multiple sequences. Put another way, subsumptive sequencing relates individual stars into a cluster and integrative sequencing relates clusters into larger constellations (Maton 2014b: 148-70).

\section{FIGURE 2}

Example of integrative sequencing (source: McGregor, 1996: 7).

Fawcett (1993) develops three major arguments against the category of VP as characterised in Halliday (1994):

(a) The clausal functional node Process (which basically indicates the action, state, or whatever, designated by the clause - Halliday 1994: 107) is vacuous if it is always and only realised by a VP. All other experiential roles are realisable by various classes of phrase - for instance, Actor and Goal may be realised by NPs or non-finite clauses.

(b) The Mood element - a combination of Subject and Finite (either first auxiliary or tense marker) according to Halliday (1994: 72) - involves elements from different ranks, phrase and word. In That woman must have read ny pnind, as Fawcett points out, the Mood element is constituted by the phrase That woman and the word must. Clearly this is an unsatisfactory state of affairs in a rank based theory (Halliday 1961). On the other hand, having the two items at the same 'layer' (though different ranks - see Huddleston 1965 on the need to differentiate the two $)^{5}$ in the clause, suggests Fawcett, is "superior as a teaching aid". 6

(c) Discontinuity in the VP is common: particles such as possibly, however and fo on may be placed between any words (almost) in a VP. For instanke, in Fawcett's example ((1) below), possibly could be placed between may and have, between have and been, or between been and eating. And in in terrogative mood the first auxiliary is not continuous with the remainder of the VP, as in Has IVy been eating the caviar?, while phrasal verbs frequently have the particle separated from the main verb, as in I phone d them $u p$.

(1) Iny nay have been eating the caviar.

None of these arguments) is compelling. As to (a), it is questionable whether it really is true that the functional role of Process - which I will henceforth refer to as SoA (State of Affairs) ${ }^{7}$ - must be realised by a VP.

In Figure 2, for example, the word-group 'these arguments' integrates a significant number of condensed meanings from many passages. The text condenses a series of passages into '(a)', '(b)' and '(c)', suggesting cohesiveness within each argument and building relations among their constitutive passages. These three arguments are then integrated and related to other meanings in the passage 'None of these arguments is compelling'. This integrative sequencing brings together multiple sequences, clauses and words into a transporter term. Such super-condensation expresses rapid building of complexity. Like subsumptive sequences, integrative sequences are often indicated by words such as 'this', 'that', 'these', etc., but are less likely to consolidate happenings into things. 
Second, horizontal sequencing can be distinguished according to what the links between passages add to their meanings:

- consequential sequencing indicates how meanings from different passages shape one another; and

- sequential sequencing indicates the ordering of meanings from different passages.

Sequential sequencing links passages and adds meanings, enabling higher epistemological condensation than segmental sequencing (see Table 2), but represents the lowest category of cumulative sequencing because it adds only temporal relations. For example, 'You have to read it and then you're asked some questions' specifies the ordering of meanings between two passages. Perhaps due to this simplicity, sequential sequencing can be repeatedly used to narrate a series of events. For example, a schoolteacher described the process of phagocytosis as: 'They find the pathogen, they engulf it, next they come around and trap it, and then they destroy it'. This creates relations between passages and adds meanings: the underlined links specify the order of actions. Linking words that often indicate sequential sequencing include 'then', 'after', 'before', 'when', 'meanwhile', etc.

Consequential sequencing goes further by adding more complex links between passages, including (in order of progressively higher epistemological condensation) causal, conditional, and counter-expectant relations. First, causal links (such as 'because' or 'so') typically involve the temporal relations of sequential sequencing but add causality, such as: 'It also has this function where it produces antibodies, cells that produces antibodies, so that the third line can act specifically'. Second, conditional links, such as 'if...then', go further than the meanings present in the passages by suggesting alternatives. For example, 'if you had a relatively low density gas being excited to emit light, then you'll get an emission spectrum' both relates meanings from the passages and creates further possible relations, such as having a high density gas and thus not getting an emission spectrum. Third, counter-expectant links add to this notion of alternative possibilities by making clear that something was indeed anticipated other than the meanings of the passages. Returning to this example:

The display of human remains on the site of Pompeii has not become a major issue even though the question of the treatment of skeletal remains has evoked impassioned debate in other parts of the world.

This indicates that the first passage was unexpected given the second and that something else was expected, resonating out to unspecified meanings. Moreover, counter-expectation additionally provides the opportunity to be combined with other consequentials, such as causal (e.g. 'even so') or conditional links (e.g. 'even if’), enabling higher epistemological condensation. 


\subsection{Subtypes of segmental sequencing}

Both cumulative and segmental sequencing add meanings; the difference is one of degree. Segmental sequencing does not explicitly link passages and so generates lower epistemological condensation. However, distinctions can be made among different kinds of segmental sequencing that express more or less building of complexity. We now outline these subtypes, beginning always from the lower category in Table 2.

Within segmental sequencing, two subtypes can be distinguished depending on whether the passages being sequenced (but not linked) are different or similar:

- compartmental sequencing brings together different passages; and

- sedimental sequencing brings together similar or identical passages.

Compartmental sequencing offers lower epistemological condensation because it simply places different passages together while sedimental sequencing adds the meaning of emphasis through repetition or reiteration. For example, in the compartmental sequencing of 'We were very pleased to open our season with a win. We made sure they couldn't run their attack', the second passage elaborates on the first by explaining how the 'win' was achieved', but the sequencing itself adds little beyond enabling this continuity of focus. Similarly, 'Okay, does that make sense? Too many notes for one day!' adds nothing beyond enabling a shift of focus. (We distinguish these two forms, below).

In contrast, sedimental sequencing both enables continuity of focus and adds emphasis, as in this exchange:

Teacher: Most of them weren't deflected at all?

Student: They weren't deflected at all.

The student's response emphasizes that the option the teacher presents is correct. Alternatively, sedimental sequencing may highlight or accentuate a specific part of the previous passage, such as: 'The Zeeman effect could not be explained. The Zeeman effect could not be explained', again adding a meaning of emphasis.

\subsubsection{Finest level of segmental sequencing}

A further level of delicacy distinguishes within compartmental and sedimental sequencing (see Table 2). First, compartmental sequencing can be distinguished into: 
- incoherent sequencing of passages with no significant relation to each other; and

- coherent sequencing of passages that offers continuity.

This explores whether sequencing that does not itself add meanings constrains or enables knowledge-building by disrupting or maintaining continuity between passages. Incoherent sequencing brings together passages that are epistemologically unrelated (though they may maintain continuity of actor or context). For example, 'The scale is red, orange, yellow, green, blue, indigo, violet. David - where's your gear?' shifts focus from scientific knowledge to classroom behaviour. The sequencing of these passages adds nothing epistemologically. Indeed, such discontinuity, typically indicated by a sudden shift in focus or topic, could be viewed as an 'absolute zero' (and potential starting point) for knowledge-building through sequencing.

In contrast, coherent sequencing expresses more opportunity for building by offering continuity, either implicitly by not shifting focus or explicitly through conjunctions such as 'and'. For example, in 'you've got a written source and you've got a picture source', the word 'and' simply signals that a further short passage will follow which remains on topic ${ }^{11}$. Similarly, the following includes two instances of coherent sequencing, one where 'and' could be inserted (underlined) and one where 'and' explicitly indicates continuity:

We're very pleased to open our season with a win. We made sure they couldn't run their attack and we managed to hold them down.

In both cases the sequencing merely affords the possibility of building meanings on a topic ${ }^{12}$.

Second, sedimental sequencing can be distinguished into:

- repetitive sequencing of passages that are practically identical; and

- reiterative sequencing of similar passages with sufficient difference to add meanings.

Repetitive sequences add nothing more that what is said in the first passage; reiterative sequences involve a discernible difference that itself adds meaning and thus slightly higher

11 The word 'and' does not necessarily indicate coherent sequencing if it is or could be followed by words such as 'so' (consequential) or 'then' (sequential).

12 Our focus here is solely on sequencing. Where coherent sequences appear to involve knowledgebuilding, this is typically achieved through clausing; here, the sequencing provides an enabling condition for, rather than an active contribution to, epistemological condensation. Accordingly, relating two different passages with 'and' represents lower epistemological condensation of sequencing than repeating a passage (sedimental sequencing). 
epistemological condensation. This could involve a change of word for a synonym, such as in: 'Something smells delicious. Yeah, something smells nice.' Although offering little extra meaning, this sequencing suggests a relation between 'delicious' and 'nice' (each could replace the other). Reiterative sequencing may also involve additional emphasis. For example, in 'Have you got your science book? Have you got your science book?', the second passage additionally highlights the significance of the emphasized term. The distinction between repetitive and reiterative sequencing is not based on whether passages are exactly identical, for repetition in spoken language often involve changes in volume, intonation, voice quality, rhythm, etc. Rather, the issue is whether any differences strengthen ESD by adding meanings. Though minimal, such additions may aid wider understanding of epistemological condensation, such as where synonymized or emphasized words are subsequently condensed or elaborated.

\section{Illustrative analyses of epistemological condensation}

The translation device outlined in Paper 1 and here offers a means for revealing the epistemic complexity of knowledge expressed through discourse. The wording and word-grouping tools of Paper 1 allocate words to relative strengths of ESD; the clausing and sequencing tools of Paper 2 allocate ways of combining words and combining passages to relative degrees of strengthening ESD or epistemological condensation. Paper 1 illustrated the wording tools through analyses of a secondary school History classroom and a scientific research abstract. In the classroom excerpt technical words were partially unpacked into everyday wording and those simpler meanings were then repacked into a technical concept, tracing a wave pattern from stronger to weaker and back up to intermediate strength of ESD (see Paper 1, Figure 4). In contrast the science abstract comprised highly technical words, often modifying each other, tracing a continuously high flatline of extremely strong ESD (Paper 1, Figure 5). These contrasting profiles of ESD raised questions of why they exhibit a wave or flatline, what drives movements of ESD up and down in the History classroom, and whether the science example involves movements of a different kind. More generally, wording analysis revealed successive states of complexity of the knowledge being expressed but not the building of complexity over time.

We shall thus briefly explore what the clausing and sequencing tools reveal about the knowledge-building expressed by discourse, using the same contrastive examples as Paper 1. For brevity, we focus on the first two levels of each tool. We retain the wording annotation from Paper 1 in our examples (see Table 4 of Paper 1 ) to enable the analyses to be related together. Obviously, space precludes repeating the wording analysis, so we strongly encourage readers to re-read section 5 of Paper 1 first. 


\section{TABLE 3}

Annotation for clausing categories

\begin{tabular}{|c|c|c|}
\hline EC & Type & Subtype \\
\hline+ & \multirow{2}{*}{ connecting } & taxonomizing \\
\hline & & coordinating \\
\hline & \multirow{2}{*}{ augmenting } & characterizing \\
\hline 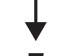 & & establishing \\
\hline
\end{tabular}

The key for annotation of clausing is shown in Table 3. All connecting has solid underlining; coordinating has no further adornment and taxonomizing has double underlining. Clausing without solid underlining is augmenting, where establishing has dashed underlining and characterizing (the most common subtype in our examples) has no underlining ${ }^{13}$.

\section{TABLE 4}

Annotation for sequencing categories

\begin{tabular}{|c|c|c|}
\hline EC & Type & Subtype \\
\hline+ & \multirow{2}{*}{$\begin{array}{l}\text { cumulative } \\
\text { (indented) }\end{array}$} & $\langle\langle$ vertical $\rangle\rangle$ \\
\hline & & $\{\{h o r i z o n t a l\}\}$ \\
\hline & \multirow{2}{*}{ segmental } & |lsedimental|| \\
\hline$\downarrow$ & & compartmental \\
\hline
\end{tabular}

The annotation key for sequencing is shown in Table 4 and uses indenting and brackets. All cumulative sequencing is indented. Stretches of horizontal sequencing (which may include

13 A 'quick-and-dirty' way to identify categories is to focus on a verb, such as in 'Pathological myopia can cause functional blindness' (coordinating-causing) or 'Pliny the Younger wrote Tacitus a letter' (characterizing-dramatizing). However, this is only a first take: the same verb may appear in multiple categories, especially variations of 'to be' ('is', 'are', 'was', etc.). 
multiple passages) are indented and identified by \{\{double curly brackets\}\} around the whole phase of sequencing and \{single curly brackets\} around links between passages. For example:

\{\{There is nothing wrong with going back to your Year 11 information on bath houses, \{because\} the activities that happened in one bath house generally happened in every other bath house.\}\}

Vertical sequencing often condenses long stretches of text, so only the final passage containing the transporter is indented with «double angle brackets» around the whole sequence and «single angle brackets s around the transporter. For example:

«Vesicles in the cytoplasm called lysosome fuse with the phagosome releasing digestive enzymes such as lysozyme and proteases into the phagosome.

The result of 〈this fusion〉 is called phagolysosome.〉〉

Segmental sequencing is not indented. Compartmental sequencing is unadorned and sedimental sequencing is identified by two vertical lines, for example: 'IIMost of them weren't deflected at all? They weren't deflected at all.|l'

\subsection{Clausing of secondary school History example}

Our first example is a teacher explaining to a Year 11 History classroom how to answer a take-home assignment in which students are asked to evaluate 'the influence of Greek and Egyptian cultures in the Roman Empire'. Wording analysis in Paper 1 revealed a wave pattern of ESD strengths: from technicals (bold) down to everydays (unadorned) and back up to technicals. This pattern is facilitated by clausing and sequencing through the excerpt. We begin by highlighting the clausing.

This is a little bit hard: "The InfLUENCE of Greek and Egyptian cultures." What does that mean? What would "the InfLUENCE of Greek and Egyptian cultures" mean, okay? No idea, right? What it means is, if we started to look at all the things in Pompeii and Herculaneum, what objects may be showing Greek design? Or Egyptian design? Or Greek mythology? Or Egyptian mythology? Or what building techniques like columns? Are there Greek col umns? Do, you know, are the themes of their artwork reflecting it? So it's saying ...remember when we started, we said that Pompeii had originally been settled by Greeks? Okay? And if we look at where Italy is, it's's not that far from Egypt at this time, umm, we've, we've had, umm ... Cleopatra has been killed by the time the volcano erupts, she ${ }^{p}$ and Mark Antony are dead and Egypt is part of the Roman Empire. So there would be massive amounts of TRADE going on, and umm, you know people visiting their diplomats you know or their, their, ambass... like their envoys, and things like that, all going back and forth across the countries. So, ideas. When you get TRADE in ideas - you wouldn't have heard this word before-we call it "aesthetic trade". Have you heard of it? Yeah. 
In short, the wave of ESD in the wording is enabled by a parallel movement from higher to lower to higher epistemological condensation in clausing, three phases we discuss in turn. In the first phase, at the start of the extract, the teacher employs relatively high epistemological condensation. After quoting the assignment topic (a word-group of stronger ESD), she asks students to unpack its meanings with two taxonomizing questions: "What does that mean? What would "the InfLUENCE of Greek and Egyptian cultures" mean, okay?'. These emphasize the stronger ESD of the topic by connecting terms into the constellations of History and, specifically, highlight the complex schemas and processes evoked by the assignment by eliciting taxonomizing from students. The teacher's questions thereby highlight the high ESD of the assignment topic and point downwards towards unpacking simpler meanings. When students fail to respond, the teacher answers herself by taxonomizing again but as a statement that begins: "What it means is....

This higher clausing sets up a second phase in which meanings condensed into the assignment topic are described by the teacher in mostly everyday wording (weaker ESD). For this unpacking the teacher moves into clausing of lower epistemological condensation: augmenting and specifically characterizing (as the lack of underlining shows, this form dominates the excerpt). She describes the actions of people (e.g. 'Pompeii had originally been settled by Greeks') and asks questions about the properties of terms (e.g. 'what objects may be showing Greek design?...'). This characterizing represents a slow but steady accumulation of meanings as the teacher enumerates ideas condensed in the essay question, augmenting each term with additional meanings. The taxonomizing of 'What it means is...' directly sets up this phase by embracing much of this characterizing. The full taxonomizing passage is:

What it means is, if we started to look at all the things in Pompeii and Herculaneum, what objects may be showing Greek design? Or Egyptian design? Or Greek mythology? Or Egyptian mythology? Or what building techniques like columns? Are there Greek columns? Do, you know, are the themes of their artwork reflecting it?

The series of characterizing clauses is thus nested within the taxonomizing. The lower clausing thereby steadily augments a series of meanings that are themselves gathered up by 'What it means is...'. The characterizing then continues until another moment of taxonomizing ('Egypt is part of the Roman Empire').

A third phase involves moving back up from simpler towards complex ideas. This is initiated and completed by two instances of taxonomizing (higher epistemological condensation). The move is signalled by 'Egypt is part of the Roman Empire', which generates a compositional taxonomy that enables subsequent meanings to be brought together. The teacher then begins the new phase by establishing a starting point ('So there would be massive amount_ _. Of IRADE going on'), accumulates meanings through characterizing actions (e.g. 'people visiting their diplomats'), before finally bringing some of these meanings together through taxono- 
mizing ('we call it "aesthetic trade"'). Here meanings previously described in everyday words (lower ESD) are condensed by the teacher into the double technical word-grouping 'aesthetic trade' (stronger ESD). As discussed in Paper I (section 5.1), until this point she uses 'TRADE' as an everyday (consolidated) word. Here, the relatively high epistemological condensation of taxonomizing condenses preceding meanings while connecting the term explicitly into History, technicalizing the wording. So this phase moves from the lowest subtype (establishing) through a higher subtype (characterizing) until reaching a highpoint (taxonomizing) that results in a technicalized term of stronger ESD.

This example suggests clausing represents a key resource for actors to articulate and develop complexity. Here, higher clausing announces the complexity of the assignment and sets up the subsequent unpacking and relating together of some of its meanings through lower clausing. These meanings are then repacked through higher clausing into a historical concept. Clausing thereby helps enable the wave profile of ESD in the wording, moving from highly complex to simpler to complex meanings.

\subsection{Sequencing of secondary school History example}

Building complexity is not confined to clausing; sequencing also helps enable movements of ESD in wording.

This is a little bit hard: "The INFLUENCE of Greek and Egyptian cultures." What does that mean? What would "the INFLUENCE of Greek and Egyptian cultures" mean, okay? No idea, right? What it means is,

$\{\{\{i f\}$ we started to look at all the things in Pompeii and Herculaneum, what objects may be showing Greek design? \{Or\} Egyptian design? \{Or\} Greek mythology? \{Or\} Egyptian mythology? \{Or\} what building techniques like columns?\}\}

Are there Greek columns?

$\{\{D o$, you know, are the themes of their artwork reflecting it?\{So\} it’s saying remember \{when\} we started, we said that Pompeii had originally been settled by Greeks? Okay?\}\}

And

$\{\{\{i f\}$ we look at where Italy is, it's's not that far from Egypt at this time, $\}\}$

umm, we've, we've had, umm ... Cleopatra has been killed by the time the volcano erupts, she ${ }^{p}$ and Mark Antony are dead and

$\{\{$ Egypt is part of the Roman Empire. \{So\} there would be massive amounts of TRADE going on, $\}\}$ 
and umm, you know people visiting their diplomats you know or their, their, ambass...like their envoys, and things like that,

$\{\{a l l$ going back and forth across the countries $\{$ So $\}$, ideas. $\}\}$

$\{\{\{$ When $\}$ you get TRADE in ideas-you wouldn't have heard this word before-we call it "aesthetic trade". $\}\}$

Have you heard of it? Yeah.

Overall, the excerpt predominantly comprises cumulative-horizontal sequencing interspersed with segmental-compartmental sequencing. The higher subtype of horizontal sequencing plays a crucial role in enabling movements in the complexity of wording from stronger to weaker ESD (phases 1-2) and from weaker to stronger ESD (phases 2-3).

First, horizontal sequencing facilitates unpacking meanings condensed within the stronger ESD of the assignment topic ('The INFLUENCE of Greek and Egyptian cultures'). For the taxonomizing of 'What it means is...', the teacher uses horizontal sequencing of passages of simpler meanings. The number of \{curly brackets\} between 'What it means is,' and 'umm, we've, we've had' show the teacher using horizontal sequencing to express conditions $\{i f\}$, alternatives $\{0 r\}$, causes $\left\{s_{0}\right\}$ and times $\{$ when\}. Each sequencing reaches out to new and potential meanings that she relates to the assignment topic using mostly everyday wording. The teacher distinguishes meanings that are condensed within the stronger ESD of the assignment into a series of different meanings expressed in simpler terms without losing sight of the whole. Here horizontal sequencing thereby enables condensed meanings to be not only unpicked and unpacked separately but also related together.

Second, horizontal sequencing contributes to moving back up towards more the complex meanings characterizing phase 3, from 'Egypt is part of the Roman Empire' onwards. The teacher relates this taxonomizing to the meanings that follow with the link $\{$ So\}. She then elaborates its implications through a series of horizontal sequences bringing together the notion that this involves trade, that trade involves movements between countries, that this movement involves ideas, and that there is 'TRADE in ideas'. Finally, this horizontal sequencing relates 'TRADE in ideas' to the higher clausing and stronger ESD wording of 'we call it "aesthetic trade"'.

This analysis suggests that sequencing represents another resource for actors to express and build complexity, one which can combine with clausing. Here the relatively high epistemological condensation of horizontal sequencing provides a means for unpacking some of the ideas condensed within complex meanings into simpler meanings and for repacking those simpler meanings into new technical wording. In both forms of packing, the ideas expressed are related through the links created among passages, building knowledge. 


\subsection{Clausing of scientific research example}

Our second example comprises the abstract for a scientific article published in Circulation (Suzuki et al., 2010: 1919). Wording analysis in Paper 1 revealed a high flatline of very strong ESD, with many technicals (bold), including many technical-conglomerates (BoLd CAPITALS). Nonetheless, the clausing and sequencing tools reveal how the text still expresses knowledgebuilding. We begin by highlighting the clausing.

Background - High-Density LIPOPROTEIN (HDL) protects the artery wall by removing cholesterol from lipid-laden macrophages. However, recent evidence suggests that hDL might also inhibit atherogenesis by combating inflammation.

Method and Results - To identify potential anti-Inflammatory mechanisms we challenged macrophages With LIPOPOLYSACCHARIDE, an inflammatory microbial ligand for Toll-like receptor 4. HDL inhibited the expression of 30\% (277 of 911) of the genes normally induced by LIPOPOLYSACCHARIDE, MICROARRAY analysis revealed. One of ITs $^{p}$ major targets was the type I interferon response pathway, a family of potent viral IMMUNOREGULATORS controlled by Toll-like receptor 4 and the TRAM/TRIF signaling pathway. Unexpectedly, the ABILITY of HDL to inhibit gene expression was independent of MACROPHAGE cholesterol stores. IMMUNOFLUORESCENT studies suggested that HDL promoted TRAM TRANSLOCATION tO INTRACELLULAR compartments, which impaired subsequent signaling by Toll-like receptor $\mathbf{4}$ and TRIF. To examine the potential in vivo relevance of the pathway, we used mice deficient in Apolipoprotein A-I, the major protein of HDL. After infection with Salmonella typhimurium, a Gram-negative bacterium that expresses LIPOPOLYSACCHARIDE, APOLIPOPROTEIN A-I-deficient mice had 6-fold higher plasma levels of interferon- $\boldsymbol{\beta}$, a key regulator of the type-I interferon response, than did wild-type mice.

Conclusions - HDL inhibits a subset of LIPOPOLYSACCHARIDE -stimulated MACROPHAGE genes that regulate the type I interferon response, and its ${ }^{P}$ ACTION is independent of sterol metabolism. These findings raise the POSSIBILITY that REGULATION Of MACROPHAGE genes by HDL might link innate immunity and CARDIOPROTECTION.

Like the classroom excerpt, the dominant category of clausing is characterizing, but here this augmenting of meanings begins from a different starting point and serves a different purpose. The History teacher used characterizing to invite students into building a consteIlation from simpler meanings. In the science abstract complex constellations are assumed as already established; the authors write for readers possessing extensive prior knowledge. In the abstract the agents whose actions and properties are being characterized are almost exclusively technicals and often conglomerates. Thus, characterizing here serves to augment meanings already possessing very strong ESD and for which rapid additional condensation is mostly unnecessary. Nonetheless, characterizing does express epistemological condensation by bringing meanings together. The clausing tool thereby highlights the knowledge-building potentially obscured if analysing only wording. 
In addition, the abstract includes three instances of connecting (underlined) that express more rapid knowledge-building. The first comprises taxonomizing of a key component of the study: 'One of Its ${ }^{p}$ major targets was the type I interferon response pathway'. This quickly tightens the focus, enabling the authors to then outline a series of complex relations discovered in researching this pathway, such as between 'the abiLITY of hDL to inhibit GeNe eXPression', 'MACrophage cholesterol stores', and 'tram translocation to intracellular compartments'. Second, at the end of 'Method and Results' the authors use taxonomizing to create two compositional relations representing results of an experiment, between 'APoLIPoprotein A-I-deficient mice' and 'wild-type mice' and their 'plasma levels of interferon- $\boldsymbol{\beta}$ '. This elaborates the results in a directly comparative manner that enables their implications to be articulated in the 'Conclusions' section. Third, the authors end with coordinating by proposing relationS-REGULATION Of MACROPHAGE genes by HDL might link innate immunity and CARDIOPROTECTION - that represents the culmination of the elaboration of meanings through the abstract and the 'take-home' message of the whole article. It is this coordinating relation that the authors suggest may become new knowledge within the intellectual field. The relatively high clausing here enables rapid epistemological condensation of meanings elaborated through the abstract and the conjecture of a highly complex idea. Rewriting terms into letters, the coordinating proposes that $A$ of $B$ by $C$ might relate $D$ and $E$, where each of these terms possess very strong ESD.

Where clausing in our pedagogic example enabled 'packing' moves between complex and simpler meanings, clausing in this research example mostly helps manage existing complexity. The authors use lower clausing to augment already very complex meanings and higher clausing at key moments to identify key elements or propose correlations among crucial meanings, creating relationships of great complexity.

\subsection{Sequencing of scientific research example}

In terms of sequencing, the abstract involves the following:

Background -

$\{\{$ High-Density LIPOPROTEIN (HDL) protects the artery wall by removing cholesterol from lipidladen macrophages. \{However\}, recent evidence suggests that HDL might also inhibit ATHEROGENESIS by combating inflammation. $\}\}$

Method and Results -

$\{\{\{T O\}$ identify potential anti-Inflammatory mechanisms we challenged maCrophages with LIPOPOLYSACCHARIDE, an inflammatory microbial ligand for Toll-like receptor 4.3$\}$

HDL inhibited the expression of 30\% (277 of 911) of the genes normally induced by LIPOPOLYSACCHARIDE, MicROARRAY analysis revealed. 
$\left\{\left\{\right.\right.$ One of ITS $^{\mathrm{P}}$ major targets was the type I interferon response pathway, a family of potent viral IMMUNOREGULATORS controlled by Toll-like receptor 4 and the TRAM/TRIF signaling pathway. $\{$ Unexpectedly\}, the ABILITY of HDL to inhibit gene expression was independent of MACROPHAGE cholesterol stores. $\}\}$

immunoflUorescent studies suggested that hDl promoted tram translocation to intracellular compartments, which impaired subsequent signaling by Toll-like receptor 4 and TRIF.

$\{\{\{T 0\}$ examine the potential in vivo relevance of the pathway, we used mice deficient in Apolipoprotein A-I, the major protein of HDL. $\}\}$

$\{\{\{A f t e r\}$ infection with Salmonella typhimurium, a Gram-negative bacterium that expresses LIPOPOLYSACCHARIDE, APOLIPOPROTEIN A-I-deficient mice had 6-fold higher plasma levels of interferon- $\boldsymbol{\beta}$, a key regulator of the type-I interferon response, than did wild-type mice. 3$\}$

COnclusions - « HDL inhibits a subset of LIPOPOLYSACCHARIDE-stimulated MACROPHAGE genes that regulate the type I interferon response, and its $^{\mathrm{p}}$ ACTION is independent of sterol metabolism.

〈These findings> raise the POSSIBILITY that REGULATION Of MACROPHAGE genes by HDL might link innate immunity and CARDIOPROTECTION. $>>$

The authors use considerable cumulative sequencing (especially horizontal sequencing) and very little segmental sequencing. Unlike the History example, most horizontal sequences comprise only two passages - there are fewer \{single curly brackets\}. The abstract offers not prolonged sequences of multiple relations but rather succinct links between adjacent meanings, reflecting the assumed knowledge of the intended audience. Here the authors can use numerous technical words without explanation, requiring fewer passages to be linked.

The authors use forms of cumulative sequencing to contextualize the paper, describe experiments and present conclusions. The 'Background' section comprises horizontal sequencing of existing knowledge in the field. This sets out the complex constellations to which the study will contribute and establishes why it will contribute by creating a tension with the link \{However\} between well-established knowledge and the possibility of changes to that received wisdom. In the 'Method and Results' section the authors use horizontal sequencing to introduce (with \{To\}) the first experiment of the study and to describe its results with the linker \{Unexpectedly\} providing a basis for conducting a second experiment. In this section, two instances of segmental (specifically, compartmental) sequencing enable further discussion of the results of the first experiment. Drawing on the sequencing tool's third level of delicacy, the specific form here is coherent sequencing, where passages are not linked but on related topics, which does not increase ESD but does enable knowledge-building by maintaining the focus of discussion. The authors then return to horizontal sequencing to introduce the second experiment (with $\{T 0\}$ ) and to describe its results, using \{After\} to relate actions 
involved in the experiment to their outcomes, including the numerous meanings condensed in clausing by the taxonomizing of two types of mice and levels of interferon- $\beta$ (see section 4.3, above). Thus, in these sections the use of horizontal sequencing enables expert readers to be rapidly brought up to speed, first on the context to and purpose of the article and then the nature of and rationale for the experiments. It also lays foundations for the abstract's conclusions, which represent a highpoint.

The whole of the 'Conclusions' section comprises vertical sequencing. Indeed, the specific form is integrative sequencing, the highest subtype for epistemological condensation: meanings are condensed from more than one passage and transported across passages. Specifically, the integrative sequencing condenses the following:

HDL inhibits a subset of LIPOPOLYSACCHARIDE-stimulated MACROPHAGE genes that regulate the type I interferon response, and its $^{p}{ }_{A C T I O N}$ is independent of sterol metabolism.

All these meanings are integrated into 〈These findings> and transported into relations with the rest of the final passage. As described above (section 4.3), that passage is relatively high clausing (coordinating) that sets up possible relations to new ideas. Thus, higher sequencing works here with higher clausing to collect together and relate the numerous, highly complex terms elaborated through the abstract.

In short, the authors employ higher sequencing to establish how the article relates to existing knowledge in the field and to show how the study may change that knowledge. This connects the research to 'before' and 'after' states of epistemological constellations, succinctly explicating its scientific contribution. Lower sequencing enables the basis of that contribution to be enumerated economically. Alongside clausing (section 4.3), this sequencing analysis also reveals that the abstract's flatline profile for wording (see Paper 1, section 5.2) does not signal stasis. Figure 3 shows the epistemological condensation exhibited by sequencing: meanings are added at different rates that trace waves through the abstract. While the wording flatline of ESD shows the discourse remains within a symbolic domain of complex constellations, the sequencing wave of epistemological condensation reveals how the ways those words are combined expresses considerable knowledge-building.

\section{Conclusion}

Discourse is devilishly difficult. Though focused solely on one form (epistemic-) of one organizing principle (semantic density) of knowledge practices, even two relatively long papers cannot embrace all attributes revealing its realizations in English discourse. As we emphasized, the translation device represents a compromise between comprehensiveness and utility. The tools offer a starting point for exploring how English discourse may express the com- 


\section{FIGURE 3}

Sequencing profile for scientific article abstract

$\mathrm{EC}+$

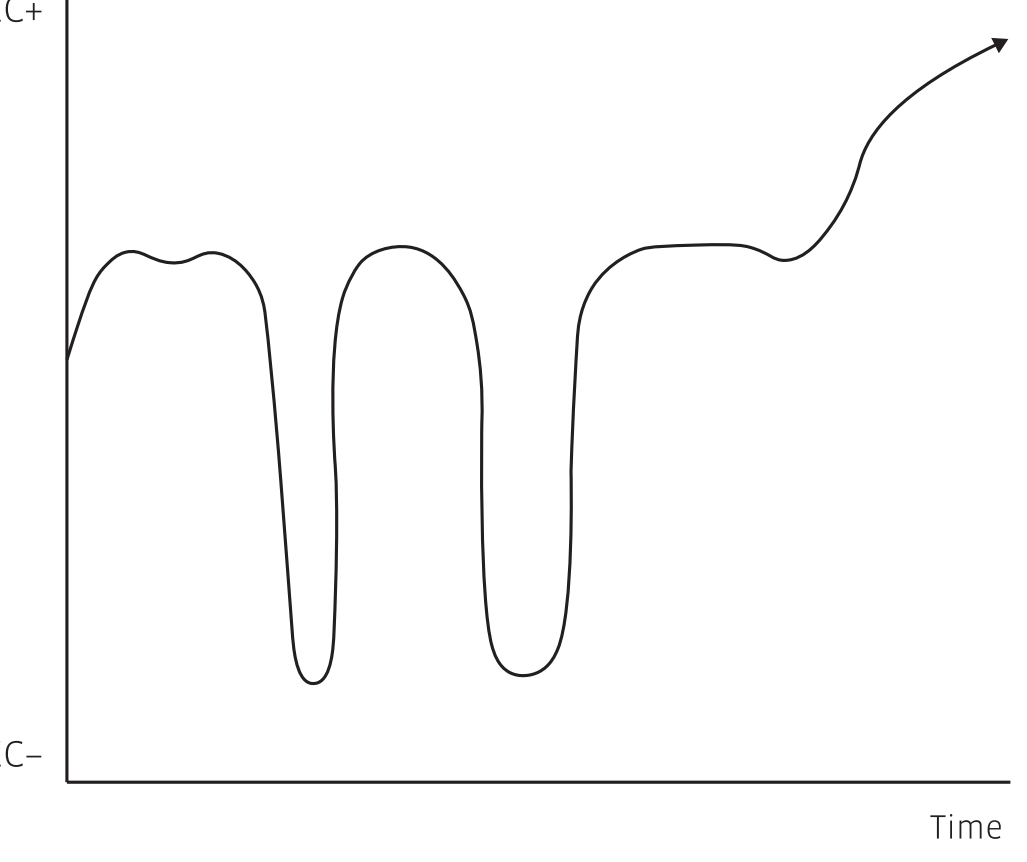

plexity of knowledge practices. The analyses in Paper 1 illustrated insights the wording and word-grouping tools provide into how ESD may be expressed. The current paper developed those analyses to illustrate insights the clausing and sequencing tools provide into how the strengthening of ESD may be expressed. In the History classroom, the clausing and sequencing used by the teacher helped facilitate waving between weaker and stronger ESD wording through unpacking and repacking meanings of terms. This weaving together of different ESD strengths contributed to introducing students to complexity. In contrast, the authors use of clausing and sequencing in the science abstract contributed to articulating and extending complexity, maintaining a high line of ESD, by establishing relations between their research and existing epistemological constellations. As the examples suggest, clausing and sequencing may each play different roles in pedagogy and research, in the humanities and sciences, or in speech and writing. Indeed, they play far more roles-our analyses barely scratch the surface of insights the translation device can offer. Nonetheless, they highlight how the four tools reveal different but complementary facets of how English discourse expresses epistemic complexity: wording and word-grouping tools explore states of complexity (ESD); and clausing and sequencing tools explore building complexity (epistemological condensation).

The translation device as a whole thereby offers a multifaceted toolkit. Which tools one uses depends on the problem-situation. This practical emphasis underpins our repeated reminders that the tools are not models of discourse but rather a translation device for enac- 
ting the concept of ESD. Thus, no specific practice, subject area or mode is intrinsically associated with a particular category of wording, clausing or sequencing. For example, it would be fundamentally mistaken to equate the humanities with everyday wording or science with technicals. The strengths of ESD and degrees of epistemological condensation are always a matter for empirical research. Moreover, in determining these features, the tools are guides for analysis, not 'cookie-cutter' rules for parsing decontextualized fragments. Enacting the tools requires judgement, immersion in one's problem-situation, and gathering data from any relevant specialized fields that can help determine, for example, whether terms are technicals or whether clausing connects a term to an epistemological constellation. We anticipate that such immersion will enable development of the translation device to embrace ways ESD is realized in English discourse that we have not captured here. Furthermore, we should reemphasize that complexity is more complex than this translation device: it does not capture ESD expressed in other languages or in other modes (such as gesture), does not translate rarefaction, does not address axiological-semantic density or other forms of complexity, and is intended to complement rather than displace the development of indicators of ESD for different practices (see Paper 1, section 1.2).

Nonetheless, ongoing studies of body movement, musical notation and images are showing that the translation device represents a valuable starting point for developing tools to translate between ESD and other discourses, modes and practices. Specifically, it sketches an analytic method for constellation analysis. As Maton (2014b: 154) previously stated:

All systems of ideas and practices - scientific, religious, political, moral, aesthetic, athletic, linguistic, etc.-comprise a semantic structure of stances chosen from a potential array, arranged into patterns, condensed with meanings, and charged with valuations. From an infinite number, all these systems identify and name particular stars (as, for example, concepts) and draw relations among them (causal, sequential, associational, compositional, etc.) to create a semantic structure of constellations.

The translation device we have outlined suggests five strategies for exploring the epistemic complexity of constellations. First, establish the principal units of ESD and epistemological condensation in the domain being studied (e.g. wording/word-grouping and clausing/sequencing). Second, identify 'Gwifflies' or solitary nodes (of which there may be several kinds) that comprise the bases for building complexity. Third, analyse ESD by identifying signs indicating the number of such nodes related together and their degree of interconnectedness. Fourth, analyse epistemological condensation by exploring how nodes are related together, how many new relations are thereby created, what form those relations take, what flows along the relations, in which directions, and so on. For these analyses, relationality, differentiation and resonance offer possible indicators for both ESD and epistemological condensation. Fifth, distinguish categories of strengths of ESD/epistemological condensation by dividing data into stronger/weaker types and, in turn, dividing those types into stronger/weaker subtypes, 
and so on. This process of recursive division enables a variety of different underlying features to be embraced.

While focused on English discourse, the translation device thereby offers suggestive directions for exploring the realizations of ESD in other domains. Such exploration is not simply epistemological. Revealing the basis of ESD is a social justice issue. Complex, sophisticated, profound, deep..., such descriptors of highly-valued practices have remained stubbornly ill-defined, obscuring the bases of achievement in social fields such as education. Their nebulous nature means only actors who already grasp their meaning by virtue of previous socialization or education can succeed. When complexity remains a black box, social inequality is encouraged. Creating translation devices that can help reveal the nature of ESD and epistemological condensation in different contexts is a step towards enabling greater social access to ways of understanding and building complexity. Thus, while conceptual, the tools we have presented in these papers are practical: their potential value lies in analysing and changing practices.

\section{Bibliographic references}

BLACKIE, M., 2014: "Creating semantic waves: Using Legitimation Code Theory as a tool to aid the teaching of chemistry", Chemistry Education Research and Practice 15, 462-9.

Clarence, S., 2014: Enabling Cumulative Knowledge-building Through Teaching: A Legitimation Code Theory analysis of pedagogic practice in law and political science, unpublished PhD thesis, Rhodes University, South Africa [available: http://www.legitimationcodetheory.com].

Macnaught, L., K. Maton, J. R. Martin \& E. Matruglio, 2013: "Jointly constructing semantic waves: Implications for teacher training", Linguistics and Education 24(1), 50-63.

Maton, K., 2009: "Cumulative and segmented learning: Exploring the role of curriculum structures in knowledge-building", British Journal of Sociology of Education 30(1), 43-57.

Maton, K., 2011a: "Knowledge-building: Analysing the cumulative development of ideas" in G. IVInson, B. Davies \& J. Fitz (eds.): Knowledge and Identity: Concepts and applications in Bernstein's sociology, London: Routledge, 23-38.

Maton, K., 2011: "Theories and things: The semantics of disciplinarity" in F. Christie \& K. Maton (eds.): Disciplinarity: Functional linguistic and sociological perspectives, London: Continuum, 62-84. Maton, K., 2013: “Making semantic waves: A key to cumulative knowledge-building”, Linguistics and Education 24(1), 8-22.

Maton, K., 2014a: "Building powerful knowledge: The significance of semantic waves" in B. BARRETT \& E. RATA (eds.): Knowledge and the Future of the Curriculum, London: Palgrave Macmillan, 181-97. 
Maton, K., 2014b: Knowledge and Knowers: Towards a realist sociology of education, London: Routledge.

Maton, K., 2016a: "Legitimation Code Theory: Building knowledge about knowledge-building" in K. Maton, S. Hood \& S. Shay (eds.): Knowledge-building: Educational studies in Legitimation Code Theory, London: Routledge, 1-24.

Maton, K., 2016b: "Starting points: Resources and architectural glossary" in K. Maton, S. Hood \& S. SHAY (eds.): Knowledge-building: Educational studies in Legitimation Code Theory, London: Routledge, 233-43.

Mcgregor, W., 1996: "Arguments for the category of verb phrase", Functions of Language 3(1), $1-30$.

Suzukı, M. et al., 2010: "High-density lipoprotein suppresses the type I interferon response, a family of potent antiviral immunoregulators, in macrophages challenged with lipopolysaccharide", Circulation 122, 1919-27. 